\title{
Behavioral Training and Performance Management of Human Services Organization Care Providers During the COVID-19 Pandemic
}

\author{
Helena Maguire ${ }^{1} \cdot$ Jill M. Harper $^{1} \cdot$ Rita M. Gardner $^{2} \cdot$ James K. Luiselli $^{1}{ }^{\circledR}$
}

Accepted: 20 December 2021 / Published online: 3 January 2022

(c) The Author(s), under exclusive licence to Springer Nature Switzerland AG 2022

\begin{abstract}
Objectives This report describes a model of training and performance management that was designed for and implemented with care providers at a residential school for children with neurodevelopmental and intellectual disabilities in response to the COVID-19 pandemic. The model focused on health and safety concerns, risk mitigation, and intervention integrity. Methods Procedures followed an applied behavior analysis (ABA) and organizational behavior management (OBM) framework. Action plans addressed (a) critical COVID-19 protocols, (b) behavior-specific implementation guidelines, (c) remote and in-person training formats, (d) in vivo supervision, and (e) knowledge and performance assessments.

Results A competency evaluation (field study) revealed that participant care providers $(N=25)$ acquired and maintained COVID-19 protocols at near $100 \%$ proficiency immediately following and 1-month post-training. Supervisors had uniformly positive approval and acceptance ratings of school responsiveness to the COVID-19 pandemic.

Conclusions Integrated and evidence-based care provider training and supervisory practices can promote risk mitigation and performance effectiveness during health crises such as the COVID-19 pandemic. More controlled research that includes multiple dependent measures is needed to replicate and extend our findings to similar human services settings.
\end{abstract}

Keywords Applied behavior analysis · Care provider training $\cdot$ COVID-19 $\cdot$ Human services organizations · Organizational behavior management $\cdot$ Performance management

The COVID-19 pandemic created and continues to impose serious problems, challenges, and obstacles for applied behavior analysis (ABA) day and residential settings serving persons with neurodevelopmental and intellectual disabilities. For example, Cox et al. (2020) published a position statement that outlined ethical dilemmas associated with suspending in-person ABA services as the result of COVID-19-related health and safety risks to clients and practitioners. They presented a decision-making model for risk mitigation during the pandemic to assist clients and their parents-guardians in determining the benefits and limitations of maintaining in-person services. Cox et al. (2020) concluded, "we strongly recommend that most in-person services be temporarily paused until public health officials

James K. Luiselli

jluiselli@melmarkne.org

1 Clinical Development and Research, Melmark New England, 461 River Road, Andover, MA 01801, USA

2 Melmark, Berwyn, PA, USA indicate a particular community, county, or state has the medical capacity to support those who are or may become seriously infected with COVID-19" (p. 302).

In response to Cox et al. (2020), LeBlanc et al. (2020) and Columbo et al. (2020) argued against a blanket interruption of ABA services. Chief among their position points were acknowledging behavioral practitioners as essential personnel during the health crisis, the regression of skills in persons with disabilities when services stop for extended periods, and commitment to the Professional and Ethical Compliance Code for Behavior Analysts which stresses continuity and non-disrupted programming with clients (BACB, 2021). The cogent and occasionally disparate analyses presented by Cox et al. (2020), LeBlanc et al. (2020), and Columbo et al. (2020) represent the highest level of ethical deliberation and problem-solving facing behavior analysts during a time of unprecedented and rapidly shifting health concerns (Bailey \& Burch, 2016).

Another consideration for ABA service settings during the COVID-19 pandemic is adherence to recommended state and federal guidelines for program operations. Kornack et al. 
(2020) highlighted several requirements such as daily health screenings, personal hygiene, wearing masks and face coverings, physical distancing, meal protocols, handling contaminated clothing, and environmental cleaning consistent with strategies recommended by the Centers for Disease Control and Prevention (Centers for Disease Control 2020). Notably, "organizations need robust staff training on adherence to the new safety procedures as well as a plan for support mechanisms that will ensure compliance" (p. 8). In particular, behavioral skills training (BST) (Parsons et al., 2012) can be adopted to instruct practitioners in best practices for health promotion and risk mitigation. As well, enhanced supervision and program monitoring will be required for performance management of the workforce (DiGennaro Reed et al. 2013; Luiselli, 2015; Reid, 2017).

Telehealth modalities have evolved within ABA (Ferguson et al., 2019; Schieltz \& Wacker, 2020; Tomlinson et al., 2018) and are another vital component of service delivery during the COVID-19 pandemic. Rodriguez (2020) detailed several steps behavior analysts can take when delivering instruction to clients through live (synchronous) videoconferencing. Two elements that constitute an effective telehealth model are assessing a client's responsiveness to skill acquisition programs and facilitating implementation integrity of care providers. Lerman et al. (2020) commented further on remote coaching of care providers via telehealth while describing several implementation barriers and solutions. For example, prior to initiating telehealth services, behavior analysts should have sufficient training in this modality, communicate expectations about interactions with clients, provide operational guidelines, confirm necessary resources (e.g., hardware, software, internet connectivity), and protect confidentiality, among other critical considerations.

Clearly, safety and risk mitigation practices when delivering in-person ABA services during the COVID-19 pandemic demand extensive training, supervision, and performance management of care providers. Organizational commitment to evidence-based methods, supportive contingencies that promote and maintain procedural compliance, and comprehensive progress monitoring are key elements of program success (Kornack et al., 2020). With regard to strategies for reducing disease transmission in the workforce, Gravina et al. (2020) reviewed several interventions with supporting evidence that behavior analysts should consider when addressing COVID-19 priorities. The procedures highlighted in their review that appear to be most effective with care providers are instructions and prompts, environmental cuing, reduced response effort, task clarifying checklists, BST, and performance feedback.

In this article, we report the design and implementation of training and performance management strategies at a specialized school for students with neurodevelopmental and intellectual disabilities necessitated by the COVID-19 pandemic. Our model identified service priorities among clients and care providers, remote and in-person training sessions comprising task analyses and instructional media, interactional guidelines for addressing high-risk behavior (e.g., aggression, spitting), in vivo supervision, and knowledge/competency-based assessments. Data from a preliminary field study are presented which illustrate the effects of training and performance management on care provider implementation of health and safety competencies. We also conducted social validity assessment of responsiveness to the COVID-19 pandemic from supervisors who were responsible for training-performance management interventions (Luiselli, 2021).

\section{Methods}

\section{Participants}

The participants were 25 care providers at a private residential school serving 140 children and youth (4 to 22 years old) with neurodevelopmental and intellectual disabilities located in the northeast region of the USA. From this group, 13 of the participants functioned as classroom instructors and 12 of the participants functioned as residential staff at six community-based group homes. All participants were 22 years of age and older and had undergraduate college degrees. We selected the participants randomly from files that contained their pre-training and post-training data which were recorded from the program described below. Thus, this was not a prospective study, there were no inclusion-exclusion criteria, and the participants formed a convenient sample.

Within classroom and group homes, the participants implemented instructional and behavior support plans with students, managed self-care and daily living routines, recorded outcome data, and performed environmental care. Classroom and group home supervisors observed in these locations and monitored direct services. After being hired at the residential school, all of the participants received preservice training that focused on educational practices, reporting guidelines, human rights, health and wellness, behavioral intervention, and other essential duties.

\section{Procedures}

School services were temporarily terminated in the third week of March 2020 after a student and several care providers tested positive for COVID-19. Following guidance from local, state, and federal agencies, school administrators concentrated on workplace assessment, safety precautions, supports to care providers, environmental modifications, and family communications. Additional areas of 
emphasis included securing personal protective equipment (PPE), establishing new maintenance routines (e.g., building cleaning and sanitizing), enforcing a no-visitation mandate, scheduling daily information and problem-solving briefings, assigning complex tasks to the most qualified supervisors, and reporting programmatic decisions to external stakeholders. Administrators also established and adhered to state-mandated guidelines for quarantine of students and employees who had a positive COVID-19 test result. Gradual transition to school services began in mid-May 2020 at which time the participants were trained on COVID-19-specific protocols and evaluated immediately following training and approximately one month later.

\section{Training}

Eight classroom supervisors and six group home supervisors conducted training with the participants assigned to those locations. The supervisors had been in their role at the residential school between 1 to 15 years $(M=6.6$ years). All of the classroom supervisors had graduate (masters) degrees and were either board-certified behavior analysts (BCBA) or certified special educators. Of the group home supervisors, all had undergraduate degrees and four of six were pursuing graduate studies. They conducted small group (2-3 persons) training sessions with the participants that were in-person, physically distanced, and mask-protected lasting 20-30 min and on occasion, delivered remotely via videoconferencing.

During sessions, supervisors reviewed one or more of eight COVID-19 protocols (Table 1) the participants were expected to follow when delivering services to students as well as interacting with co-workers. Each protocol was task analyzed into component steps that were descriptive and easy to understand (Russ et al., 2013). In illustration, Table 2 shows the task analysis created for putting on and removing $\mathrm{N}-95$ masks. As seen in this example, task analyses not only

Table 1 COVID-19 protocols

\begin{tabular}{|c|c|c|}
\hline Competency & General description & $\begin{array}{l}\text { Number of } \\
\text { checklist } \\
\text { steps }\end{array}$ \\
\hline Entering school building & Apply face mask, enter building, wash hands, follow directional arrows, take-record temperature & 8 \\
\hline Changing clothing-outerwear & Remove-place soiled clothing in garment bag, double bag, wash hands, apply new outerwear & 9 \\
\hline Cleaning_-disinfecting & $\begin{array}{l}\text { Gather materials, put on gloves, apply solution-wipe-dry designated surfaces, return materials, } \\
\text { remove-discard gloves }\end{array}$ & 9 \\
\hline Infrared thermometer & Place, align, hold, trigger, and read thermometer display at forehead level & 6 \\
\hline PPE-N-95 mask & Place, align, secure, remove, place mask in designated area & 9 \\
\hline PPE_face shield & Place, strap, remove, sanitize, and return face shield to designated area & 6 \\
\hline PPE_-gloves & Wash hands, place-remove gloves on both hands, discard gloves in designated area & 14 \\
\hline Washing hands & Wet, lather, scrub, rinse. and dry hands for designated time period & 11 \\
\hline
\end{tabular}

Table 2 Task analyses: putting on and removing N-95 mask

Putting on

Steps

1: Hold mask in hand and place firmly over mouth, nose, and chin

2: Stretch and position bottom band under ears

3: Stretch and position top band high at back of head

4: Press thin metal wire along upper edge against bridge of nose to fit securely on face

5: Perform a fit check by breathing in and out—when breathing out, check Mask covers mouth, nose, and chin secured by bottom and top bands for air leakage around face

\section{Removing}

Steps

6: Remove bottom band by touching only the band and bringing it carefully over head

7: Grasp top band and bring it carefully over head

8: Pull mask away from face without touching front

9: Place mask in designated area
Step completion

Mask covers mouth, nose, and chin

Bottom band is located under ears

Top band is located at back of head

Mask covers mouth, nose, and chin secured by bottom and top bands

Step completion

Bottom strap is located over head

Top (and bottom) bands are located over head

Mask is removed and held by band in hands

Mask is located in designated area 
defined procedural steps but also described what each step should look like when completed correctly.

Supervisors reviewed the COVID-19 protocol task analyses with accompanying PowerPoint slides that featured (a) written procedural descriptions and instructions, (b) diagrams and picture illustrations of performing task analysis steps correctly, and (c) additional video demonstrations of competent performance. Participants role-played each protocol, rehearsed the procedures that would apply with students and co-workers, and received behavior-contingent praise and correction from the supervisors conducting training sessions. These combined methods of instruction, demonstration, rehearsal, and feedback were consistent with behavioral skills training (BST) shown to be effective with human services employees (Belisle et al., 2016; Reid, 2017; Whiting et al., 2014).

Participants were required to complete online training assignments for each of the COVID-19 protocols. There was a quiz per learning assignment they had to pass with $100 \%$ accuracy before advancing to the next assignment. Quiz items included multiple choice options, true-false statements, and open responses to questions. Immediately following training sessions, participants were evaluated by demonstrating the protocol they had been trained on while the supervisor completed an observational behavior checklist (see the "Measures" section). A performance score less than $100 \%$ produced further training until the full competency was achieved.

\section{Performance Management}

Supervisors conducted post-training performance management by observing the participants in vivo during their routine implementation of the COVID-19 protocols. They used the same behavior checklists to monitor and evaluate the participants during these observations. Post-observation, supervisors delivered feedback with descriptive praise following accurately implemented steps and correction of any steps that were misapplied. Supervisors who were appropriately masked and physically distanced completed in vivo performance management with the participants 1-2 times per week such that all of the COVID-19 protocols were evaluated every month. Similar to their initial training, observations with performance feedback continued until participants achieved a $100 \%$ accuracy score on each critical competency.

\section{Measures}

\section{Competency Evaluations}

We designed behavior checklists that corresponded to the steps outlined in each of the eight COVID-19 protocols. For example, Fig. 1 is the behavior checklist for putting on and removing gloves. During the immediate post-training and 1-month post-training observations, supervisors scored the checklists by recording each step the participants completed correctly and incorrectly summarized as a percent accuracy score.

\section{Social Validity Assessment}

Social validity assessment was conducted with the 14 classroom and group home supervisors from a five-item questionnaire: (1) The school effectively communicated decisions about responding to the COVID-19 pandemic, (2) The school implemented plans that kept students and staff safe and healthy during the COVID-19 pandemic, (3) The training I received helped me supervise staff follow COVID-19 protocols, (4) I was able to conduct supervision confidently and efficiently during the COVID-19 pandemic, and (5) I would recommend the training I received and my supervision responsibilities during the COVID-19 pandemic to other human services programs. We distributed the questionnaire to supervisors via an electronic survey platform that they completed anonymously and returned by a deadline date (return rate $=100 \%$ ).

\section{Data Analyses}

Analysis of the competency evaluation data consisted of computing the average percent of behavior checklist steps the participants completed correctly (correct steps/correct plus incorrect steps $\times 100$ ) on each COVID-19 protocol with the exception of entering the school building which applied only to participants who were classroom instructors. Social validity assessment data were analyzed by computing the average rating for each questionnaire item rated by the 14 supervisors (sum of numerical ratings per item/number of supervisors).

\section{Results}

Figure 2 shows the average percent of behavior checklist steps the participants completed correctly on each COVID19 protocol immediately following training sessions and during in vivo observations approximately 1 month later. Individual participants responded with uniform proficiency within a range of 86.6 to $100 \%$ correct. Average scores per protocol were between 97.6 and $100 \%$ at the immediate posttraining and 1-month post-training phases.

Table 3 presents the rank-ordered average ratings for each item on the social validity questionnaire. The highest average rating was that the school implemented plans to address the safety and health of students and staff $(M=4.78)$. All 
Fig. 1 Behavior checklist: putting on and removing PPEgloves

\begin{tabular}{|c|c|c|}
\hline \multicolumn{3}{|l|}{ PUTTING ON } \\
\hline Steps & $\begin{array}{l}\text { Completed } \\
\text { Correctly }\end{array}$ & $\begin{array}{l}\text { Completed } \\
\text { Incorrectly }\end{array}$ \\
\hline 1: Wash hands or apply hand sanitizer & & \\
\hline 2: Remove glove from dispenser & & \\
\hline 3: Check gloves for rips/defects & & \\
\hline 4: Open glove cuff, insert hand, move fingers to ensure snug fit & & \\
\hline 5: Roll top of glove down to cuff on wrist & & \\
\hline 6: If wearing gown, roll glove cuff over end of sleeves & & \\
\hline 7: Repeat steps 2-6 with other hand & & \\
\hline REMOVING & & \\
\hline Steps & $\begin{array}{l}\text { Completed } \\
\text { Correctly }\end{array}$ & $\begin{array}{l}\text { Completed } \\
\text { Incorrectly }\end{array}$ \\
\hline 8: Take one gloved hand and grasp outer edge near wrist area & & \\
\hline 9: Fold over and pin away from hand & & \\
\hline 10: Turn inside out and remove glove & & \\
\hline 11: Hold removed glove with gloved hand & & \\
\hline 12: Place bare fingers inside cuff of worn glove without touching exterior & & \\
\hline $\begin{array}{l}\text { 13: Pin off from inside, turning inside out, removing glove and envelop with } \\
\text { other glove }\end{array}$ & & \\
\hline 14: Discard gloves in designed area & & \\
\hline
\end{tabular}

Percent Accuracy Score (correct steps/correct + incorrect steps x 100) other questionnaire items also received an average "agree" rating related to supervisor training and recommendation to other human services settings $(M=4.42)$, school communication about responding to the COVID-19 pandemic $(M=4.21)$, and conducting supervision confidently and efficiently $(M=4.14)$.

The data in Table 4 are the results of COVID-19 surveillance testing initiated at the school in March 2020 through September 2021. Relatively low incidence rates among employees were documented during early months, which increased in November 2020, December 2020, and January 2021 and subsequently declined in the remaining months of the reporting period. Within the student population, COVID19-positive tests were consistently stable between 0 and 3 cases each month.

\section{Discussion}

The COVID-19 pandemic significantly impacted human services settings in many ways, most notably posing health and safety risks to children and adults with IDD who may also be medically fragile, display challenging behavior, and have difficulty following virus prevention guidelines. Further, care providers confronted risks when delivering services within congregate living environments due to threats of virus exposure, transmission when in close contact with clients, and implementing procedures such as physical prompting and response interruption. Relative to the work described in this article, Gardner et al. (2020) advised that, "The need to care for those with developmental disabilities with cooccurring medical fragility and/or behavioral challenges in a safe environment necessitated an immediate, structured, and thoughtful organizational response" (p. 33). Thompson and Nygren (2020) proposed the same precautions in discussing the urgency of maintaining services to persons with intellectual and developmental disabilities while protecting them and their care providers from the COVID-19 virus.

As described herein, the setting for this field study designed, implemented, and evaluated several care provider training and performance management interventions to address safety and risk mitigation objectives caused by the COVID-19 pandemic. The basis of these initiatives was to establish policies and procedures that were governed by health priorities consistent with state and federal recommendations, applied school-wide, and capable of being 
Fig. 2 Average percent of behavior checklist steps completed correctly by classroom participants $(N=13)$ (top panel) and group home participants $(N=12)$ (bottom panel) per COVID-19 protocol

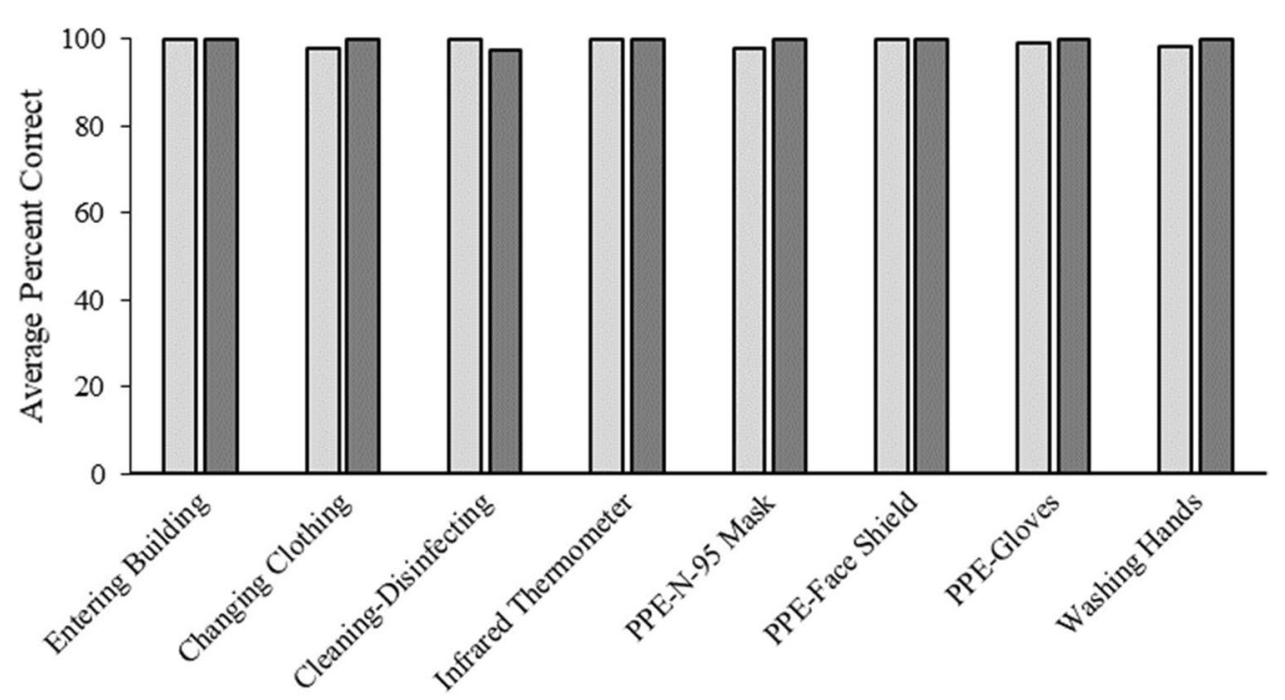

口Immediate Post-Training $\quad$ One-Month Post-Training

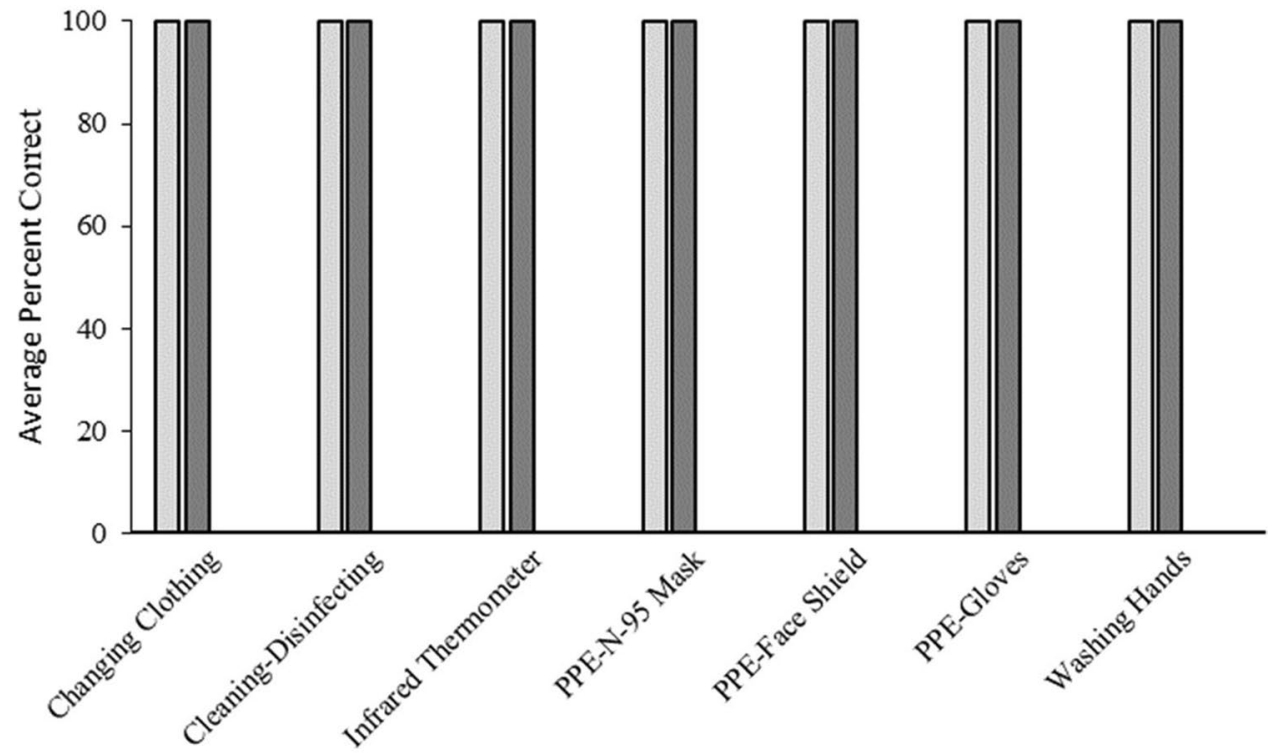

Table 3 Rank-ordered average ratings of items in social validity questionnaire

\begin{tabular}{lc}
\hline Questionnaire item & Average rating \\
\hline The school implemented plans that kept students and staff safe and healthy during the COVID-19 pandemic & 4.78 (range: $4.0-5.0$ ) \\
The training I received helped me supervise staff follow COVID-19 protocols & 4.42 (range: $4.00-5.00$ ) \\
I would recommend the training I received and my supervision responsibilities during the COVID-19 pandemic to & 4.42 (range: 3.00-5.00) \\
other human services settings & 4.21 (range: 1.00-5.00) \\
The school effectively communicated decisions about responding to the COVID-19 pandemic & 4.14 (range: 3.00-5.00) \\
I was able to conduct supervision confidently and efficiently during the COVID-19 pandemic
\end{tabular}

1 , strongly disagree; 2 , disagree; 3 , neither disagree nor agree; 4 , agree; 5 , strongly agree

implemented with integrity. Specifically, we relied on task analyzed behavior checklists, virtual and in-person BST, in vivo supervision, performance feedback, and knowledge and competency evaluations as the foundations of a comprehensive strategy toward risk mitigation. These methods were selected because they have robust evidence-based support, 
Table 4 COVID-19 surveillance testing results

\begin{tabular}{lll}
\hline Month & $\begin{array}{l}\text { Positive test results: } \\
\text { employees }\end{array}$ & $\begin{array}{l}\text { Positive } \\
\text { test results: } \\
\text { students }\end{array}$ \\
\hline March 2020 & 39 & 1 \\
April 2020 & 18 & 7 \\
May 2020 & 1 & 0 \\
June 2020 & 1 & 0 \\
July 2020 & 2 & 0 \\
August 2020 & 1 & 0 \\
September 2020 & 6 & 0 \\
October 2020 & 6 & 1 \\
November 2020 & 12 & 0 \\
December 2020 & 17 & 0 \\
January 2021 & 10 & 3 \\
February 2021 & 1 & 0 \\
March 2021 & 2 & 0 \\
April 2021 & 0 & 0 \\
May 2021 & 0 & 0 \\
June 2021 & 0 & 0 \\
July 2021 & 2 & 0 \\
August 2021 & 6 & 3 \\
September 2021 & 1 & 1 \\
\hline
\end{tabular}

have been replicated many times within human services organizations, and recommended to teach and maintain care provider skills (DiGennaro Reed et al., 2013; Lerman et al., 2015; Parsons et al., 2012, 2013; Reid, 2017).

We note that our model of training and performance management evolved over many years of systems development and evaluation devoted to contemporary practices with persons who have neurodevelopmental and intellectual disabilities (Luiselli et al., 2021), making it possible to shift rapidly in response to the novel demands of the COVID-19 pandemic. School administrators were able to quickly enact steps for purchasing PPE, modifying the physical environment, sustaining employee salaries, and paying overtime compensation, among other fiscal necessities. Second, many of the training and performance management objectives and procedures emerged from behavioral research dedicated to general health promotion. For example, Casella et al. (2010) reported on the positive effects of reduced response effort for increasing glove-wearing and hand-sanitizing by therapists at an autism treatment center. Carr et al. (2013) implemented training and graphic performance feedback with autism services care providers that improved environmental cleaning of treatment rooms. More recently, Jess and Dozier (2020) summarized antecedent and consequence strategies for teaching and maintaining hand-washing among children. Thus, many interventions are clearly applicable for dealing with essential COVID-19 health practices and can likely be extended to behaviors such as wearing face coverings, donning and doffing PPE, and physical distancing (Gravina et al., 2020).

Our competency evaluation revealed that participants responded effectively to training and performance management that was delivered virtually and in-person, notwithstanding several limitations and procedural constraints. For example, measurement according to behavior checklists was conducted with participants immediately following training sessions and 1 month later but absent in baseline phase. Pretraining measures, we determined, would be artificially low because the participants had no prior experience or received instruction for implementing the novel protocols which were designed in response to the extraordinary challenges posed by the COVID-19 pandemic. As well, training and performance management procedures were delivered as an "intervention package" that did not analyze the influence of separate components. Finally, our evaluation included a relatively small sample of participants although representative of the larger population of classroom and group home care providers at the school.

The COVID-19 infection rates among students and employees decreased contemporaneously with implementation of training and performance management strategies. These critical health data also demonstrated greater containment of the virus compared to regional incidence reporting (Massachusetts Department of Public Health COVID-19 Dashboard, n.d.). Though speculative, the increased cases reported in the period November 2020 through January 2021 may have reflected greater exposure occasioned by vacation and holiday contacts but with contained transmission among students. The relationship between school responsiveness to the COVID-19 pandemic and positive test results is purely correlational but demonstrates a desirable outcome that supports large-scale risk mitigation. At the time of this writing and in conjunction with students and care providers being fully vaccinated, virus infection rates remain low. Also, social validity assessment found uniformly positive ratings among the supervisors who implemented training and performance management with participants. The findings demonstrated favorable reactions to leadership decisions about health and safety plans, training guidance for supervising COVID-19 protocols, and conducting supervision effectively.

\section{Limitations and Future Research Directions}

Within the context of a field study, the competency evaluation was conducted to facilitate rapid and practical assessment of the outcomes from training and performance management but did not conform to the most controlled research methodology. In this regard, inter-observer agreement (IOA) was not recorded during measurement, and supervisor 
interactions with participants were not checked for implementation integrity. Many uncontrolled factors such as the familiarity of participants with supervisors plus their prior training experiences could have contributed to evaluation outcomes. Hence, research-to-practice translation of the procedures we used will be strengthened through evaluation designs that have sufficient experimental rigor to support large-scale application within human services organizations (Culig et al. 2005; Kazdin, 2011).

Further, the field study was restricted to a single setting and client population. In addition to limits of generalization, another qualification is that some programs may be challenged by implementation barriers such as lack of personnel resources and funds to administer training and performance management with full integrity. However, it is a priority for organizations educating and treating persons with intellectual, developmental, and related disabilities to implement a comprehensive and dedicated response to crises such as the COVID-19 pandemic that is informed by evidence-based methods and translated effectively at the level of service provision. Therefore, performance diagnostic assessment (Wilder et al., 2020) of obstacles to safety and risk mitigation plans is another recommended research objective.

Author Contribution HM, JMH, RMG, and JKL contributed equally to the design and evaluation of training and performance management procedures, data analysis, and writing of the manuscript.

\section{Declarations}

Ethics Approval All procedures were approved by senior administration at the residential school and conformed to the 1964 Helsinki Declaration and its later amendments or comparable ethical standards.

Consent to Participate All individuals participating in this program description consented to the training, supervisory, and evaluation procedures.

Conflict of Interest The authors declare no competing interests.

\section{References}

Bailey, J. S., \& Burch, M. R. (2016). Ethics for behavior analysts (3rd edition). Routledge.

Behavior Analyst Certification Board (2021). Professional and ethical compliance code for behavior analysts. Littleton, $\mathrm{CO}$.

Belisle, J., Rowsey, K. E., \& Dixon, M. R. (2016). The use of in situ behavioral skills training to improve staff implementation of the PEAK Relational Training System. Journal of Organizational Behavior Management, 36, 71-79. https://doi.org/10.1007/ s40614-017-0119-4

Carr, J. E., Wilder, D. A., Majdalany, L., Mathisen, D., \& Strain, L. A. (2013). An assessment-based solution to a human service employee performance problem. Behavior Analysis in Practice, 6(1), 16-32. https://doi.org/10.1007/BF03391789

Casella, S. E., Wilder, D. A., Neidert, P., Rey, C., Compton, M., \& Chong, I. (2010). The effects of response effort on safe performance by therapists at an autism treatment facility. Journal of Applied Behavior Analysis, 43, 729-734. https://doi.org/10. 1901/jaba.2010.43-729

Centers for Disease Control. (2020). Guidance for child care programs that remain open. Retrieved from https://www.cdc.gov/ coronavirus/2019-ncov/community/schools-childcare/guidancefor-childcare.html.

Columbo, R. A., Wallace, M., \& Taylor, R. (2020). An essential service decision model for applied behavior analytic providers during crisis. Behavior Analysis in Practice. Published online: 20 May 2020. https://doi.org/10.1007/s40617-020-00432-z

Cox, D. J., Plavnick, J. B., \& Brodhead, M. T. (2020). A proposed process for risk mitigation during the COVID-19 pandemic. Behavior Analysis in Practice, 13, 299-305. https://doi.org/10. 1007/s40617-020-00430-1

Culig, K. M., Dickinson, A. M., McGee, H. M., \& Austin, J. (2005). An objective comparison of applied behavior analysis and organizational behavior management research. Journal of Organizational Behavior Management, 25(1), 35-72. https:// doi.org/10.1300/J075v25n01_02

DiGennaro Reed, F. D., Hirst, J. M., \& Howard, V. J. (2013). Empirically supported staff selection, training, and management strategies. In D. D. Reed, F. D. DiGennaro Reed, \& J. K. Luiselli (Eds.), Handbook of crisis intervention and developmental disabilities (pp. 71-85). New York: Springer.

Ferguson, J., Craig, E. A., \& Dounavi, K. (2019). Telehealth as a model for providing behavior analytic interventions to individuals with autism spectrum disorder: A systematic review. Journal of Autism and Developmental Disorders, 49(2), 582-616. https://doi.org/10.1007/s10803-018-3724-5

Gardner, R. M., Maguire, H., Quigley, S. P., \& Weiss, M. J. (2020). Ensuring best outcomes for children with behavioral needs and adults with medical fragility in a congregate care setting amid the COVID-19 pandemic. Behavioral Health News, 8, 15-17.

Gravina, N., Nastasi, J. A., Sleiman, A. A., Matey, N., \& Simmons, D. E. (2020). Behavioral strategies for reducing disease transmission in the workplace. Journal of Applied Behavior Analysis, 53(4), 1-20. https://doi.org/10.1002/jaba.779

Jess, R. L., \& Dozier, C. L. (2020). Increasing handwashing in young children: A brief review. Journal of Applied Behavior Analysis, 53(3), 1219-1224. https://doi.org/10.1002/jaba.732

Kazdin, A. E. (2011). Single-case research designs: Methods for clinical and applied settings (2nd edition). Oxford University Press.

Kornack, J., Williams, A. L., Johnson, K. A., \& Mendes, E. M. (2020). Reopening the doors to center-based ABA services: Clinical and safety protocols during COVID-19. Behavior Analysis in Practice. Published online: 30 June 2020. https://doi.org/10.1007/ s40617-020-00462-7

LeBlanc, L. A., Lazo-Pearson, J. F., Pollard, J. S., \& Unumb, L. S. (2020). The role of compassion and ethics in decision making regarding access to applied behavior analysis services during the COVID-19 crisis: A response to Cox, Plavnick, and Brodhead. Behavior Analysis in Practice. Published online: 16 June 2020. https://doi.org/10.1007/s40617-020-00446-7

Lerman, D. C., LeBlanc, L. A., \& Valentino, A. L. (2015). Evidencebased application of staff and caregiver training procedures. In H. Roane, J. E. Ringdahl, \& T. Falcomata (Eds.), Clinical and organizational applications of applied behavior analysis (pp. 321-351.). Elsevier.

Lerman, D. C., O'Brien, M. J., Neely, L., Call, N. A., Tsami, L., Schieltz, K. M., Berg, W. K., Graber, J., Huang, P., Kopelman, T., \& Cooper-Brown, L. J. (2020). Remote coaching of caregivers via 
telehealth; challenges and potential solutions. Journal of Behavioral Education. Published online: 01 April 2020. https://doi.org/ 10.1007/s10864-020-09378-2

Luiselli, J. K. (2015). Performance management and staff preparation. In F. D. DiGennaro \& D. D. Reed (Eds.), Autism service delivery: Bridging the gap between science and practice in autism service delivery (pp. 465-489). Springer.

Luiselli, J. K. (2021). Performance management interventions. In J. K. Luiselli, R. M. Gardner, F. L. Bird, \& H. Maguire (Eds.), Organizational behavior management for intellectual and developmental disabilities (pp. 100-123). Routledge.

Luiselli, J. K., Gardner, R. M., Bird, F. L., \& Maguire, H. (2021). Organizational behavior management approaches for intellectual and developmental disabilities. Routledge.

Massachusetts Department of Public Health COVID-19 Dashboard. Retrieved Dec 9, 2021 from https://www.mass.gov

Parsons, M. B., Rollyson, J. H., \& Reid, D. H. (2012). Evidence-based staff training: A guide for practitioners. Behavior Analysis in Practice, 5, 2-11. https://doi.org/10.1007/BF03391819

Parsons, M. B., Rollyson, J. H., \& Reid, D. H. (2013). Teaching practitioners to conduct behavioral skills training: A pyramidal approach for training multiple human service staff. Behavior Analysis in Practice, 6, 4-16. https://doi.org/10.1007/BF03391798

Reid, D. H. (2017). Competency-based staff training. In J. K. Luiselli (Ed.), Applied behavior analysis advanced guidebook: A manual for professional practice (pp. 21-40). Elsevier/Academic Press.

Rodriguez, K. A. (2020). Maintaining treatment integrity in the face of a crisis: A treatment selection model for transitioning direct ABA services to telehealth. Behavior Analysis in Practice, 13, 291-298. https://doi.org/10.1007/s40617-020-00429-8

Russ, S., Rout, S., Sevdalis, N., Moorthy, K., Darzi, A., \& Vincent, C. (2013). Do safety checklists improve teamwork and communication in the operating room? A Systematic Review. Annals of Surgery, 258(6), 856-881. https://doi.org/10.1097/sla. 0000000000000206

Schieltz, K. M., \& Wacker, D. P. (2020). Functional assessment and function-based treatment delivered via telehealth: A brief summary. Journal of Applied Behavior Analysis, 53(3), 1242-1258. https://doi.org/10.1002/jaba.742

Thompson, J. R., \& Nygren, M. A. (2020). COVID-19 and the field of intellectual and developmental disabilities: Where have we been? Where are we? Where do we go? Intellectual and Developmental Disabilities, 58(4), 257-261. https://doi.org/10.1352/1934-955658.4.257

Tomlinson, S. R. L., Gore, N., \& McGill, P. (2018). Training individuals to implement applied behavior analysis procedures via telehealth: A systematic review of the literature. Journal of Behavioral Education, 27, 172-222. https://doi.org/10.1007/ s10864-018-9292-0

Whiting, S. W., Miller, J. M., Hensel, A. M., Dixon, M. R., \& Szekely, S. (2014). Increasing the accuracy of EpiPen administration with a brief behavioral skills training package in a school for autism. Journal of Organizational Behavior Management, 34(4), 265278. https://doi.org/10.1080/01608061.2014.973632

Wilder, D. A., Cymbal, D., \& Villacorta, J. (2020). The performance diagnostic checklist-human services: A brief review. Journal of Applied Behavior Analysis, 53, 1170-1176. https://doi.org/10. 1002/jaba.676

Publisher's Note Springer Nature remains neutral with regard to jurisdictional claims in published maps and institutional affiliations. 